\title{
A new direction for grid cells
}

\author{
Ken Cheng ${ }^{1}$
}

Published online: 21 October 2015

(C) Psychonomic Society, Inc. 2015

Summary Krupic, Bauza, Burton, Barry, \& O'Keefe (Grid cell symmetry is shaped by environmental geometry. Nature, $518,232-235,2015)$ found that the grids of grid cells in the entorhinal cortex of rats become sparser at the sharp end of a trapezoidal space. This makes grids, and grid cells, unsuitable for measuring distance traveled (odometry) and suggests that grid cells may have more to do with sorting out directions.

Place cells in the hippocampus of rodents fire most at one or a number of places in the environment. Grid cells in the entorhinal cortex fire at a regular triangular grid of locations. In the dorsomedial to ventrolateral direction, the grid size increases systematically. Collections of neighboring grid cells have similar orientations in space but are shifted slightly in the locations they cover. In 2014, this work on the neurophysiology of spatial cognition in rodents won the Nobel Prize in Physiology or Medicine for John O'Keefe, for his work on place cells (half the prize), and Edvard and Mai-Britt Moser, for their work on grid cells (the other half). This Outlook concerns a recent work from O'Keefe's group on grid cells (Krupic, Bauza, Burton, Barry \& O'Keefe, 2015), which has already drawn some commentaries (e.g., Jeffery, 2015).

Two proposed functions for grid cells are the coding of places and a metric for odometry (the measurement of distance traveled; Jeffery, 2015). In the place coding scheme, one place and one place cell correspond to a conjunction of the firing of many grid cells, with grids at different scales. If,

\footnotetext{
Ken Cheng

ken.cheng@mq.edu.au
}

1 Department of Biological Sciences, Macquarie University, Sydney NSW 2109, Australia for example, five grid cells firing together define a place, then few places satisfy those conditions, and the next conjunction may be too far away to be relevant. According to the metric hypothesis, a regular grid works like a graph paper for measuring distances, irrespective of what places are where-an abstract Cartesian grid for metric space, albeit with triangles rather than squares.

Krupic et al.'s (2015) results put the odometry hypothesis into some doubt. First, they found that grid orientation can follow the arena itself rather than the extra-arena cues around the room. Grid cells were recorded before and after a square arena was rotated by $45^{\circ}$. They shifted accordingly by an amount not significantly different from $45^{\circ}$. Grid cells from different modules of neighboring cells also maintained the same relative orientations to one another across geometric transformations of the arena (between square, hexagonal, and circular shapes). Perhaps the most startling result was that in an irregular space, grid spacing was irregular.

The irregular grids were found in a trapezoidal arena in which one end of the long (principal) axis was narrower than the other end. The grid at the narrow end was a good deal sparser (see Fig. 1a) and might also be slightly "bent." This means that if a regular grid were used as a metric, the metric would be distorted. This was expressed in Jeffery's (Jeffery, 2015) comment, titled "Distorting the Metric Fabric of the Cognitive Map." Jeffery suggested that the distortion would be small, although her comment ends by being quite open about other possibilities.

My thoughts are that these results, together with other recent findings, suggest that grid cells may serve a primary function in coding direction rather than odometry or defining a place. The maintenance of relative angular difference between modules of grid cells (Krupic et al., 2015) suggests a role in keeping track of directions. Input from cells that code head direction, namely, head-direction cells, seems to be 
a

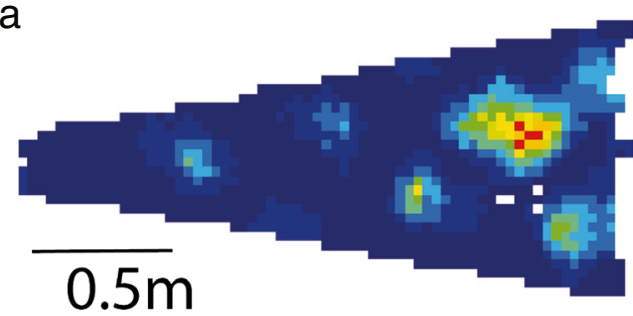

b

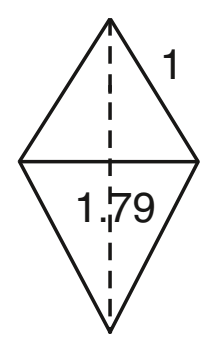

C

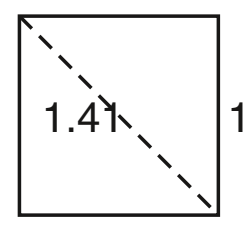

Fig. 1 a One grid cell's firing in a trapezoidal space from Krupic et al.'s (2015) Figure 3b. Warmer colors indicate higher rates of firing. $\mathbf{b}$ and $\mathbf{c}$. If keeping in a direction means keeping to a grid line (solid lines), the traveler needs to distinguish the intervertex distances along solid lines from off-course travel directions such as moving along the dotted lines. For a triangular grid (b), the ratio of the intervertex distance in the mistaken direction (along the dotted line) to the correct intervertex distance is 1.79 . For a square grid, the equivalent ratio is only 1.41 , making the distinction more difficult. Note. a from "Grid Cell Symmetry Is Shaped by Environmental Geometry," by J. Krupic, M. Bauza, S. Burton, C. Barry, and J. O'Keefe, 2015, Nature, 518, pp. 232-235. Reprinted with permission from the authors and from Macmillan Publishers Ltd.

necessary for the grid-like properties of grid cells (Winter, Clark \& Taube, 2015). Disrupting the input of headdirection cells from the anterior thalamic nucleus to the entorhinal cortex (using anesthetics) disrupted the grid properties of grid cells. Krupic et al. (2015) also found that the axes of the grids were aligned close to the direction along walls but most often were offset by a small amount. Such offsets might serve a role in disambiguating the geometrically confusing sides of the walls in a square arena, another role in sorting out directions. In geometrically regular arenas, rats and their head-direction cells both succumb sometimes to geometric ambiguity (Golob, Stackman, Wong \& Taube, 2001). Golob et al. found that the rotational errors of the head-direction cells, however, have no correlation with errors made by the rats themselves. It would be interesting to see if grid cells misalign (e.g., grid rotated by $90^{\circ}$ in a square) and whether such misalignments predict the rat's rotational errors.

One possible function for grid cells is amassing information from multiple head-direction cells to increase directional precision. Any single head-direction cell has a sharp-looking peak but fires over a range of directions. Pooling multiple head-direction cells should increase precision. This hypothetical pooling may be akin to the pooling of many sensory neurons that are sensitive to polarized light in insects for telling direction with a sky compass, a process that also increases precision.

The periodic firing of relevant grid cells might tell the animal that it is keeping in the desired direction. Traveling along some path of shortest intervertex distances might allow the animal to maintain the correct direction. A triangular grid, however it is produced, packs efficiently the coding of six different directions into one cell. To keep on path, the traveler must distinguish intervertex distances and keep to the shortest intervertex distances for a collection of grid cells. When the intervertex distance is longer than expected, the traveler is off course. It is easier to tell such off-course travel from on-course travel with a triangular grid than with a square grid (see Fig. $1 \mathrm{~b}$ and c), perhaps a reason for the triangular tessellation of grids. It matters little if the grid is sparser at narrow ends of spaces because the directions of travel at these regions are limited.

It is likely that grid cells, and the entorhinal cortex, play multiple roles in keeping track of direction. Thus, some cells in the human entorhinal cortex respond selectively to clockwise versus anticlockwise direction of travel around a virtual block on a monitor (Jacobs, Kahana, Ekstrom, Mollison \& Fried, 2010), another kind of directional code.

And odometry? That can be accomplished if the entorhinal cortex has cells sensitive to the speed at which the animal is moving, increasing firing rate with speed of movement, with distance traveled calculated as speed $\times$ time. Such "speed cells," as they are called, have recently been discovered in the other Nobel Prize-winning lab (Kropff, Carmichael, Moser \& Moser, 2015).

In short, Krupic et al.'s (2015) data show that the grids of grid cells are not always regular, throwing some doubt on their role in odometry. The import of my proposal is that perhaps grid cells have less or nothing to do with odometry or place coding but serve directional coding in a number of ways.

\section{References}

Golob, E. J., Stackman, R. W., Wong, A. C., \& Taube, J. S. (2001). On the behavioural significance of the head direction cells: Neural and behavioural dynamics during spatial memory tasks. Behavioral Neuroscience, 115, 285-304.

Jacobs, J., Kahana, M. J., Ekstrom, A. D., Mollison, M. V., \& Fried, I. (2010). A sense of direction in human entorhinal cortex. Proceedings of the National Academy of Sciences USA, 107, 6487-6492.

Jeffery, K. J. (2015). Distorting the metric fabric of the cognitive map. Trends in Cognitive Sciences, 19, 300-301.

Kropff, E., Carmichael, J. E., Moser, M.-B., \& Moser, E. I. (2015). Speed cells in the medial entorhinal cortex. Nature, 523, 419-424.

Krupic, J., Bauza, M., Burton, S., Barry, C., \& O'Keefe, J. (2015). Grid cell symmetry is shaped by environmental geometry. Nature, 518, 232-235.

Winter, S. S., Clark, B. J., \& Taube, J. S. (2015). Disruption of the head direction cell network impairs parahippocampal grid cell signal. Science, 347, 870-874. 